\title{
Regional Difference in Colonic Motility Response to Electrical Field Stimulation in Guinea Pig
}

\author{
Jung Myun Kwak, ${ }^{1,2}$ Reji Babygirija, ${ }^{1}$ Irena Gribovskaja-Rupp, ${ }^{1}$ Toku Takahashi, ${ }^{1 *}$ Shigeru Yamato ${ }^{3}$ and Kirk Ludwig ${ }^{1}$ \\ ${ }^{1}$ Department of Surgery, Medical College of Wisconsin and Zablocki VA Medical Center, Milwaukee, WI, USA; ${ }^{2}$ Department of Surgery, College \\ of Medicine, Korea University, Seoul, Korea; and ${ }^{3}$ Division of Gastroenterology, National Center of Neurology and Psychiatry, Tokyo, Japan
}

\section{Background/Aims}

In isolated guinea-pig colon, we investigated regional differences in peristalsis evoked by intrinsic electrical nerve stimulation.

\section{Methods}

Four colonic segments from mid and distal colon of Hartley guinea pigs, were mounted horizontally in an organ bath. Measurement of pellet propulsion time, intraluminal pressure, electrical field stimulation (EFS; $0.5 \mathrm{~ms}, 60 \mathrm{~V}, 10 \mathrm{~Hz}$ ), and response of pharmacological antagonists, were performed to isolated segments of colon to determine the mechanisms underlying peristaltic reflexes evoked by focal electrical nerve stimuli.

Results

In fecal pellet propulsion study, the velocity of pellet propulsion was significantly faster in the distal colon and decreased gradually to the proximal part of the mid colon. Intraluminal pressure recording studies showed that luminal infusion initiated normal peristaltic contractions (PCS) in $82 \%$ trials of the distal colon, compared to that of mid colon. In response to EFS, the incidence of PCs was significantly increased in the distal colon in contrast, the incidence of non-peristaltic contractions (NPCs) was significantly higher in the middle-mid colon, distal-mid colon and distal colon, compared to that of proximal-mid colon. Addition of L-NAME into the bath increased the frequency of NPCs. EFS failed to cause any PCs or NPCs contractions in the presence of hexamethonium, atropine or tetrodotoxin.

\section{Conclusions}

This study has revealed that electrical nerve stimulation of distal colon is the most likely region to elicit a peristaltic wave, compared with the mid or proximal colon. Our findings suggest that EFS-evoked PCs can be modulated by endogenous nitric oxide.

(J Neurogastroenterol Motil 2013;19:192-203)

\section{Key Words}

Anti-peristalsis; Colonic transit; Nitric oxide

Received: November 6, 2012 Revised: February 21, 2013 Accepted: March 3, 2013

(c) This is an Open Access article distributed under the terms of the Creative Commons Attribution Non-Commercial License (http://creativecommons. org/licenses/by-nc/3.0) which permits unrestricted non-commercial use, distribution, and reproduction in any medium, provided the original work is properly cited.

*Correspondence: Toku Takahashi, MD, PhD

Financial support: None.

Department of Surgery, Zablocki VA Medical Center, 5000 West National Avenue, Milwaukee, WI 53295, USA

Tel: +1-414-384-2000 (ext. 41472), Fax: +1-414-382-5374, E-mail: ttakahashi@mcw.edu

Conflicts of interest: None.

Author contributions: Toku Takahashi designed the research. Jung Myun Kwak and Reji Babygirija performed the experiments and analyzed data. Jung Myun Kwak, Reji Babygirija and Toku Takahashi wrote the manuscript. Jung Myun Kwak, Irena Gribovskaja-Rupp, Reji Babygirija, Toku Takahashi and Kirk Ludwig contributed critical revision. 


\section{Introduction}

Migrating motor complexes (MMCs) exhibited during the interdigestive state in upper gastrointestinal tract are comprised of periodic groupings of phasic contractions in humans, ${ }^{1}$ dogs, ${ }^{2}$ rats $^{3}$ and mice despite the lack of intraluminal content. In contrast, colon always has luminal content even in the interdigestive state and exhibits a diverse range of motor patterns suited for particular physiological functions. Non-propagating colonic contractions are presumed to serve segmenting or mixing functions. High amplitude propagating contractions (HAPCs) give rise to powerful luminal occlusive contractions that can last a long time and propagate exclusively anally to ensure mass movement of feces over long distances. ${ }^{5-7}$

Colonic migrating motor complexes (CMMCs) were defined when the contractile activities propagated more than half the length of the colon in the oral or anal direction in dogs in vivo. ${ }^{8-10}$ All other patterns of occurrence of contractile activities are called colonic non-migrating motor complexes (CNMCs). ${ }^{8}$ In between the occurrence of CMMCs, CNMCs were frequently observed in dogs. ${ }^{8}$ In rats, only $9 \%$ of propagating bursts of activity were found to propagate over the full length of colon. The occurrence of shortly propagated bursts in the oral or anal directions was $29 \%$, while the stationary bursts accounted for $62 \%$ of overall bursts in rats in vivo. ${ }^{11}$

Although all segments of the colon can show various contractile activities to carry out their roles to mix, store and propel feces, as well as absorb fluids, it is well known that there are distinct functional variations among different portions of the colon. The role of the proximal colon is to mix and store liquid feces, favoring the absorption of excess water and electrolytes and forming dehydrated feces. ${ }^{12}$ In contrast, distal colon serves mainly to expel the fecal material and exhibits a pattern of intense peristaltic movements. ${ }^{13,14}$ The combination of these regional variations facilitates balance among diverse colonic functions.

Guinea pigs have been widely used to study regional difference of colonic motility. ${ }^{15,16}$ However, there are few literature sources that investigate regional variations in terms of colonic motor patterns. Although some common characteristics and pathways of enteric nervous system are preserved across vertebrates, major inter-species as well as inter-regional differences in the chemical coding, ${ }^{17,18}$ electrophysiological properties ${ }^{19}$ and receptors for peptide neurotransmitters of enteric neurons ${ }^{20}$ exist.

The electrical field stimulation (EFS) has been widely used to investigate the role of enteric nervous system in mediating gastrointestinal motility. However, there are few demonstrations that EFS evokes peristalsis. Previous studies showed that EFS failed to initiate peristalsis in the rat ${ }^{21}$ and dog colon. ${ }^{22}$ In contrast, when EFS was applied sequentially from the proximal to distal colon, aborad contractions were observed in the dog distal colon. ${ }^{22}$ Even in normal conditions, shortly propagated bursts in the oral directions, anti-peristalsis, are observed in the rat ${ }^{11}$ and $\operatorname{dog}$ colon $^{8}$ in vivo. However, the mechanism of anti-peristalsis still remains unclear. It has been shown that anti-peristalsis is observed when electrical stimulation is applied sequentially in the dog distal colon. ${ }^{22}$

To understand relationships between the motor patterns and putative functions of the colon, it is necessary to clarify the region-specific features and characteristics of motor patterns. We investigated the regional differences of the colonic motor patterns in guinea pigs in vitro. The aim of the present study was to investigate the mechanism of anti-peristalsis, by the application of EFS at oral or anal ends of colonic preparations and the of the guinea pig distal colon.

\section{Materials and Methods}

\section{Animals}

Animals were maintained and handled in accordance with policies of the Animal Care and Use Committee of Clement J. Zablocki VA Medical Center (Milwaukee, WI, USA). All the experimental protocols were approved by same institution and in accordance with the National Institutes of Health Guide for the Care and Use of Laboratory Animals.

Male Hartley guinea pigs (body weight 300-350 g) were obtained from Charles River Laboratories (Durham, NC, USA). The animals were housed in a controlled environment $\left(21 \pm 1^{\circ} \mathrm{C}\right.$, 50-70\% humidity, 12-hour light dark cycle) and given free access to food and water. They were housed at standard conditions for at least 7 days prior to any experimentation.

\section{Fecal Pellet Propulsion Study In Vitro}

Animals were euthanized by isoflurane (5\%). The entire colon was rapidly removed and four different colonic segments (proximal portion of the middle colon, middle portion of the middle colon, distal portion of the middle colon and distal colon) were taken. The middle colon was defined from the point that contained formed feces down to the level of lower pole of the left 
kidney. The distal colon was excised from the lower pole of the left kidney to the pelvic brim (Fig. 1A). The proximal colon, which contained liquid non-formed feces, was excluded because the lumen is too large to perform pellet propulsion study.

Colonic segments ( $6 \mathrm{~cm}$ in length) were laid horizontally in the organ bath filled with Krebs-Henseleit buffer (KHB; $\mathrm{MgSO}_{4} 1.2 \mathrm{mM}, \mathrm{KH}_{2} \mathrm{PO}_{4} 1.2 \mathrm{mM}, \mathrm{KCl} 4.8 \mathrm{mM}, \mathrm{NaCl} 118$ $\mathrm{mM}, \mathrm{NaHCO}_{3} 25 \mathrm{mM}, \mathrm{CaCl}_{2} 2.5 \mathrm{mM}$ and D-glucose $11 \mathrm{mM}$ ) at $37^{\circ} \mathrm{C}$ and bubbled with $95 \% \mathrm{O}_{2}$ and $5 \% \mathrm{CO}_{2}$. The colonic segments were fixed to the bottom of the bath. After 1 hour equilibration, a natural fecal pellet $(4 \mathrm{~mm}$ in diameter and $10 \mathrm{~mm}$ in length) coated with nail-polish was inserted into the oral end. The pellet propulsion was measured by monitoring the time it took for the pellet to traverse the colonic segments. The studies were repeated 5-10 times at 5 minute-intervals.

\section{Intraluminal Pressure Recording In Vitro}

As previously reported, ${ }^{23,24}$ colonic segments ( $6 \mathrm{~cm}$ in length) were laid horizontally in the organ bath filled with KHB. The oral end of the segment was connected to an infusion syringe, and the anal end was connected to a pressure transducer (AD Instruments, Colorado Springs, CO, USA). After equilibration of 30 minutes, $0.3 \mathrm{~mL} \mathrm{KHB}$ was slowly infused for 30 seconds through the oral end. The colonic segments were kept distended with KHB for 10 minutes. Then, luminal content was removed and tissues were allowed 20 minutes for equilibration without distension. Similar distention studies were repeated 5-7 times. We have recently shown that $0.7 \mathrm{~mL}$ of luminal infusion was needed to initiate motor responses with concomitant increase of intraluminal pressure in rats. ${ }^{24}$ In contrast, our preliminary study showed that $0.3 \mathrm{~mL}$ of luminal infusion was able to initiate motor responses with concomitant increase of intraluminal pressure in guinea pigs. Motor responses and intraluminal pressure changes were consistent at least 5-7 times in each tissue in response to luminal infusion. Intraluminal pressure changes in response to luminal infusion were recorded by a software system (Power-Lab model $8 \mathrm{SP}$; AD Instruments). Motor responses to luminal infusion were recorded. If peristaltic waves propagated across at least half of the colonic segment, this motor response was defined as a normal peristaltic contraction (PC). In response to luminal distension, various types of motor responses were observed, such as localized contractions, or partially propagated contractions. These motor responses were defined as non peristaltic contractions (NPCs). The frequency (the number of contractions per minute), the amplitude (difference between the baseline and the peak of the intraluminal pressure) and the motility index ( $\mathrm{MI}$; area under the curve expressed as $\mathrm{cmH}_{2} \mathrm{O} \cdot \mathrm{sec}$ ) were analyzed and regional differences were compared.

\section{Electrical Field Stimulation}

Colonic segments (middle colon and distal colon) were stimulated by electricity (EFS; $0.5 \mathrm{~ms}, 60 \mathrm{~V}, 10 \mathrm{~Hz}$ for $2 \mathrm{sec}-$ onds) during the luminal distension. Two parallel platinum electrodes separated by $0.5 \mathrm{~cm}$ were placed on the oral end of colonic segment. EFS was applied 15-20 seconds after finishing the spontaneous peristaltic reflex of the distal portion of the middle colon and distal colon. EFS was applied 5 min after luminal distension of the proximal portion of the middle colon and the middle portion of the middle colon. EFS at the oral end was applied twice during luminal infusion. EFS was repeated for each luminal infusion (5-7 times).

The electrodes were placed on the anal end of the distal colon for EFS. EFS at the oral (oral-EFS) and anal ends (anal-EFS)

\section{A}

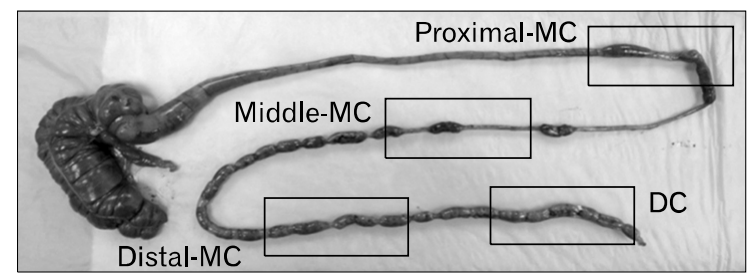

B

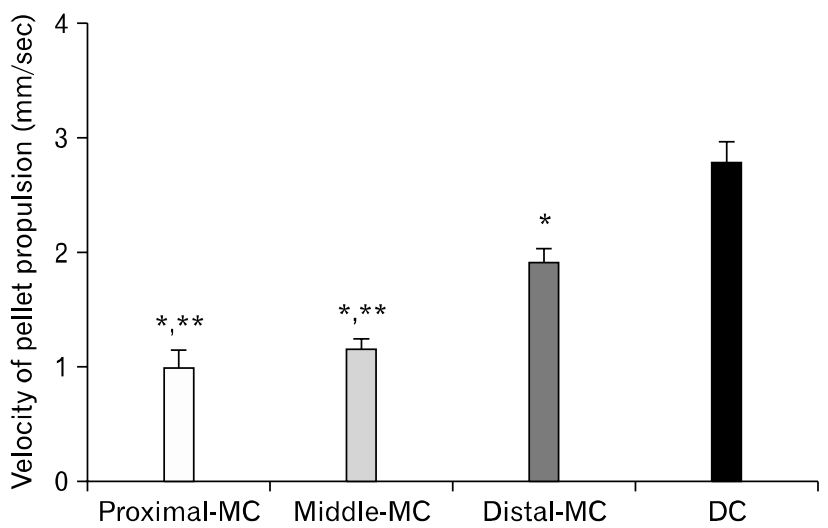

Figure 1. Gross anatomy of the guinea pig colon (A) and regional differences of fecal pellet propulsion among of the 4 different segments (B). The velocity of pellet propulsion was significantly higher in the distal colon and reduced at the proximal part of the mid colon. MC, mid colon; DC, distal colon. ${ }^{*} P<0.05$ vs. DC, ${ }^{*} P<0.05$ vs. distal-MC, $\mathrm{n}=5$. 
was applied once during luminal infusion in random order. EFS was repeated for each luminal infusion (5-7 times).

\section{Chemicals}

NG-nitro-L-arginine methyl ester (L-NAME) was purchased from Tocris Bioscience (Bristol, UK). Atropine, hexamethonium and tetrodotoxin (TTX) were obtained from Sigma Chemical Company (St. Louis, MN, USA). L-NAME $\left(10^{-4} \mathrm{M}\right)$, atropine $\left(10^{-6} \mathrm{M}\right)$, hexamethonium $\left(10^{-4} \mathrm{M}\right)$ and $\operatorname{TTX}\left(10^{-6} \mathrm{M}\right)$ were applied to the bath 20 minutes prior to luminal infusion. The doses of L-NAME, TTX, atropine and hexamethonium were selected based on the previous reports. ${ }^{14,25-27}$

\section{Statistical Methods}

Data were shown as means \pm SE. Student's $t$ test was used to compare differences in continuous variables between the 2 groups. An analysis of variance with post-hoc Duncan test was used to compare the regional differences of the colon. Statistical significance was defined as $P<0.05$.

\section{Results}

\section{Fecal Pellet Propulsion Study}

In the proximal portion of the middle colon fecal pellet propulsion occurred in 30 of 50 trials performed in 5 tissues. In the remaining 20 trials, the pellet stopped in the middle of the segment in 7 trials, and in 13 trials the pellet failed to propagate altogether. The middle portion of the middle colon showed complete pellet expulsion in $90 \%$ of 50 trials but failed to initiate propulsion in 5 trials performed in 5 tissues. In the distal portion of the middle colon and distal colon, the fecal pellet expelled within 10 minutes in all the trials tested. No retrograde propagation of the pellet was observed in any colonic tissue. The velocity of pellet propulsion was significantly higher in the distal colon $(2.8 \pm 0.6$ $\mathrm{mm} / \mathrm{sec}$ ) and reduced in the proximal part of the middle colon (Fig. 1B).

\section{Intraluminal Pressure Recording}

Recording of intraluminal pressure changes in four different segments of the guinea pig colon revealed typical features of motor response. In response to luminal infusion $(0.3 \mathrm{~mL})$, a high

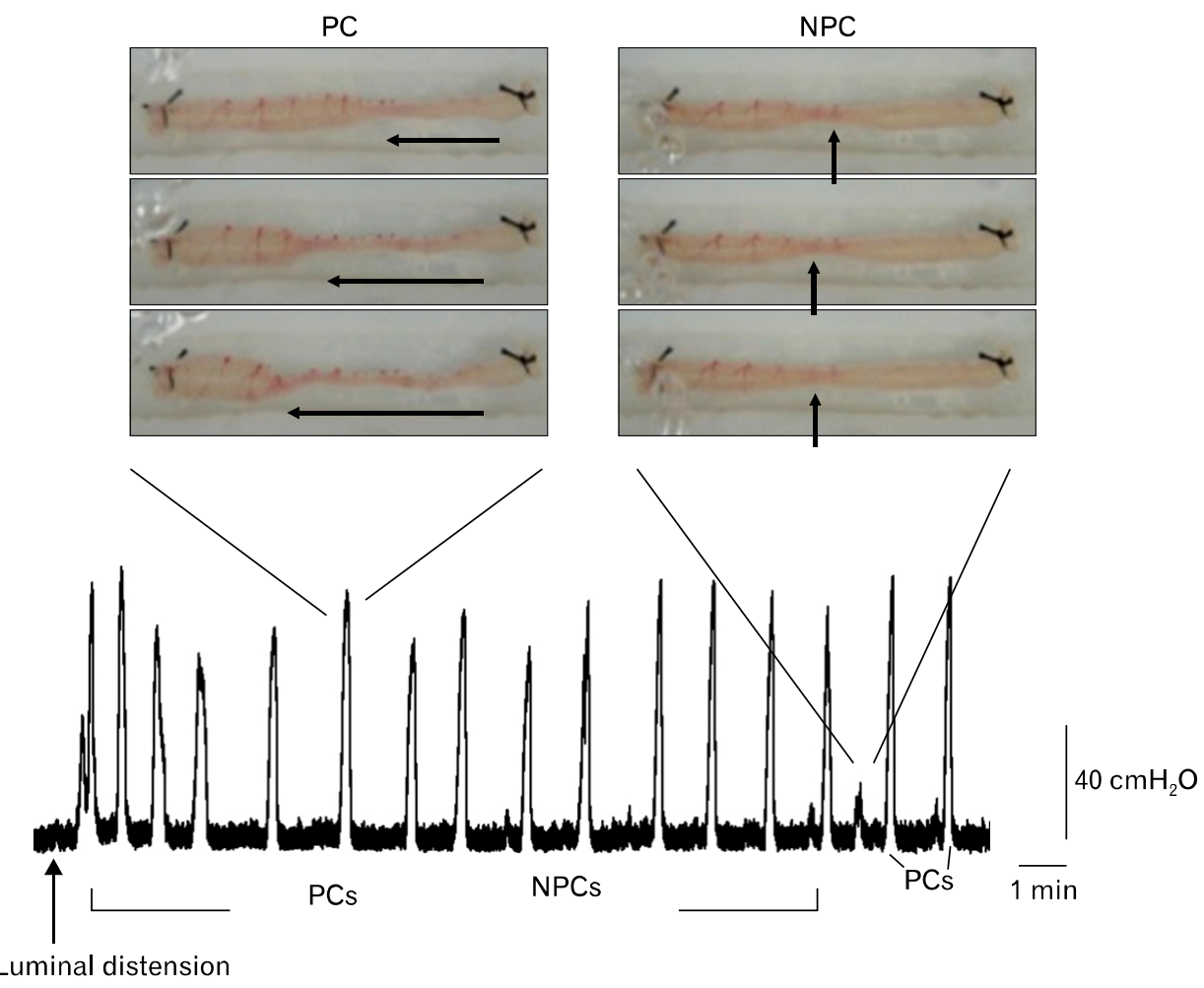

Figure 2. Intraluminal pressure increase and motor responses to luminal distension of the distal colon. In response to luminal infusion $(0.3 \mathrm{~mL})$, pressure increase was observed in the distal colon. The incidence of peristaltic contractions (PCs; $81.5 \pm 7.0 \%, \mathrm{n}=6$ ) was higher than that of non-peristaltic contractions (NPCs; $18.5 \pm 7.0 \%, \mathrm{n}=6$ ). Vertical arrows indicate NPCs, while horizontal arrows indicate PCs. 
pressure increase $\left(40-80 \mathrm{cmH}_{2} \mathrm{O}\right)$ was observed in the distal colon (Fig. 2). During luminal distension, pressure increase was spontaneously observed every 90-100 seconds. The pressure increase was highly associated with the peristaltic movement. The rate of PCs was $81.5 \pm 7.0 \%$ and the incidence of NPCs was $18.5 \pm 7.0 \%$ in 130 trials of 6 tissues of the guinea pig distal colon (Fig. 2).

In response to luminal infusion $(0.3 \mathrm{~mL})$, the proximal portion of the middle colon showed phasic or irregular NPCs rather than PCs. In contrast, the more distal part of the middle colon showed the more regular and frequent PCs and a lower incidence of NPCs (Table and Fig. 3).

The mean amplitude of overall contractions was highest in the distal colon $\left(78.6 \pm 2.8 \mathrm{mH}_{2} \mathrm{O}\right)$ and decreased gradually to the proximal part of the mid colon $\left(32.5 \pm 1.2 \mathrm{mH}_{2} \mathrm{O}, P<\right.$ 0.05 ) (Fig. 3). When compared to the distal colon, the distal-mid colon showed similar MI. however, MI was significantly decreased in the proximal-mid colon $(55.9 \pm 4.9 \%$ of the distal colon, $P<0.01)$ and the middle-mid colon $(75.1 \pm 7.9 \%$ of the distal colon, $P<0.05)$ (Table).

Table. Motility Patterns in Response to Luminal Infusion of the Different Regions of the Guinea Pig Colon

\begin{tabular}{lcccc}
\hline & $\begin{array}{c}\text { Frequency of PCs } \\
(\text { events/min) }\end{array}$ & $\begin{array}{c}\text { Frequency of NPCs } \\
(\text { events/min })\end{array}$ & $\begin{array}{c}\text { Amplitude of PCs and NPCs } \\
\left(\mathrm{cmH}_{2} \mathrm{O}\right)\end{array}$ & $\begin{array}{c}\text { Motility index } \\
\left(\mathrm{cmH} \mathrm{H}_{2} \mathrm{O} \cdot \mathrm{sec}\right)\end{array}$ \\
\hline Proximal-MC $(\mathrm{n}=6)$ & $0.16 \pm 0.09^{\mathrm{a}}$ & $1.20 \pm 0.37^{\mathrm{a}}$ & $32.5 \pm 1.2^{\mathrm{a}}$ & $5332 \pm 420^{\mathrm{a}}$ \\
Middle-MC $(\mathrm{n}=6)$ & $0.54 \pm 0.08$ & $0.25 \pm 0.06$ & $46.9 \pm 1.9^{\mathrm{a}}$ & $7053 \pm 737^{\mathrm{a}}$ \\
Distal-MC $(\mathrm{n}=8)$ & $0.47 \pm 0.05$ & $0.30 \pm 0.11$ & $49.6 \pm 2.2^{\mathrm{a}}$ & $9105 \pm 370$ \\
DC $(\mathrm{n}=6)$ & $0.60 \pm 0.05$ & $0.19 \pm 0.09$ & $78.6 \pm 2.8$ & $9591 \pm 501$
\end{tabular}

Data were obtained from 150-200 cases of peristaltic contractions (PCs) and non-peristaltic contractions (NPCs) from 6-8 tissues. MC, middle colon; DC, distal colon. ${ }^{\mathrm{a}} P<0.05$ vs. DC.

A

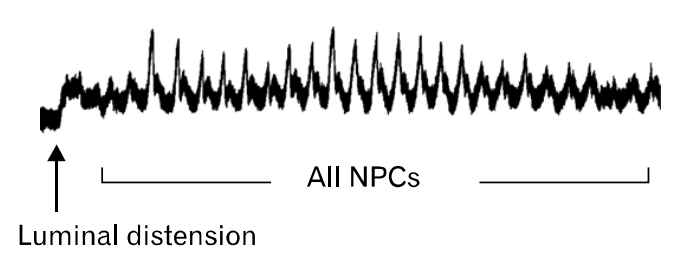

C

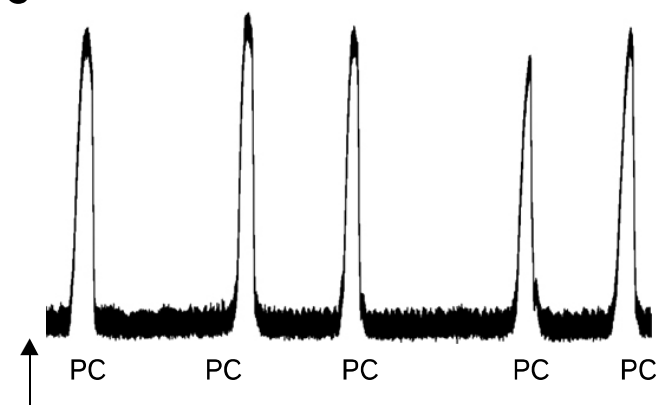

Luminal distension
B

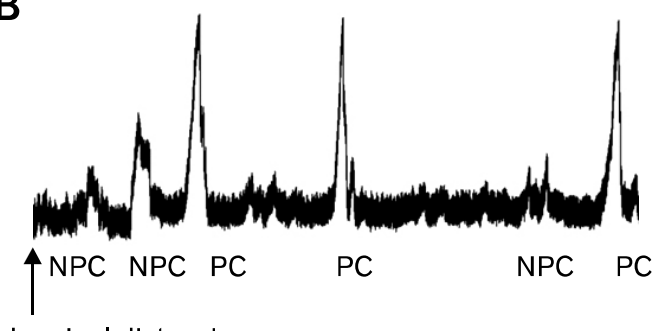

Luminal distension

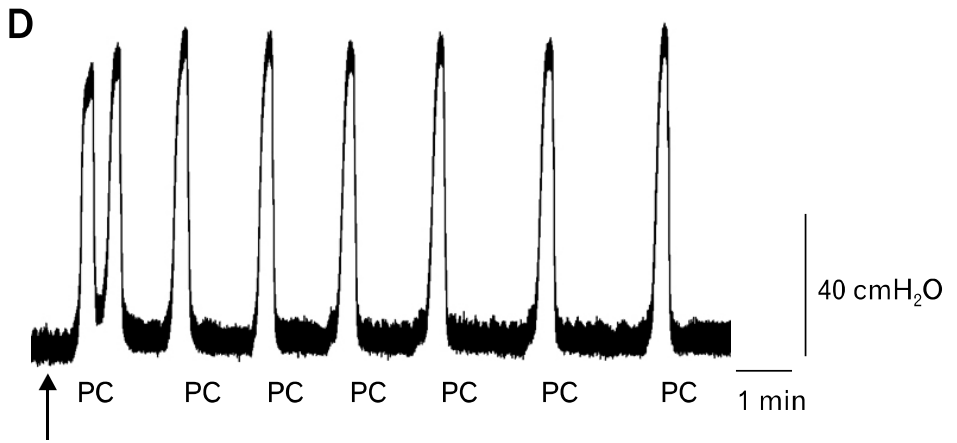

Luminal distension

Figure 3. Regional differences of motility patterns in response to luminal distension. Motor responses of the proximal portion of the mid colon (A), middle portion of the mid colon (B), distal portion of the mid colon (C) and distal colon (D) are shown. The proximal-mid colon showed frequent phasic contractions (A), while the middle-mid colon showed a mixed pattern of phasic contractions, non-peristaltic contractions (NPCs) and peristaltic contractions (PCs) (B). The distal-mid colon and distal colon showed regular and frequent PCs with less incidence of NPCs (C and D). 


\section{Motor Response to Electrical Field Stimulation}

EFS was applied at the oral end (oral-EFS) of the 4 colonic segments. When EFS was applied after spontaneous PC, EFS was not able to evoke $\mathrm{PC}$ at the proximal-mid colon and middle-mid colon (Fig. 4A and 4B). In contrast, EFS evoked PCs at the distal-mid colon and distal colon (Fig. 4C and 4D). In response to oral-EFS, a significant increase of luminal pressure concomitant with $\mathrm{PC}$ was observed in $74.7 \pm 11.5 \%$ trials in the distal colon (Fig. 4D and 4E). In contrast, NPCs were observed in $13.7 \pm 9.0 \%$ trials in response to EFS and no responses to EFS was observed in $11.7 \pm 7.3 \%$ of the distal colon (48 preparations, $\mathrm{n}=6$ ).

The incidence of EFS-evoked PCs tended to increase in the middle-mid colon and distal-mid colon $(60 \%$ in response to 50 EFSs, $n=6)$, compared to that of proximal mid colon ( $30 \%$ in response to 48 EFSs, $\mathrm{n}=6$ ).

The incidence of PCs was significantly increased in the distal colon ( $82 \%$ in response to $50 \mathrm{EFSs}, \mathrm{n}=6$ ), compared to that of the proximal-mid colon ( $30 \%$ in response to $48 \mathrm{EFSs}, \mathrm{n}=6$ ). In contast, the incidence of NPCs was significantly lower in the middle-mid colon, distal-mid colon and distal colon, compared to that of proximal-mid colon (Fig. 4) (45-60 preparations, $n=6$ ).

When EFS was applied at the anal end (anal-EFS), a significant increase of luminal pressure increase concomitant with PC was also observed in $48.9 \pm 9.2 \%$ in the distal colon (Fig. 5A). The incidence of PCs in response to EFS was not significantly different between the oral and anal stimulation (Fig. 5A). NPCs and no responses were observed in $35.9 \pm 5.2 \%$ and $15.2 \pm$ $7.4 \%$, respectively, in response to anal-EFS. The incidence of NPCs in response to anal-EFS was significantly higher $(35 \%$ in response to 50 EFSs, $\mathrm{n}=6$ ) than that of oral-EFS ( $15 \%$ in re-

$A$

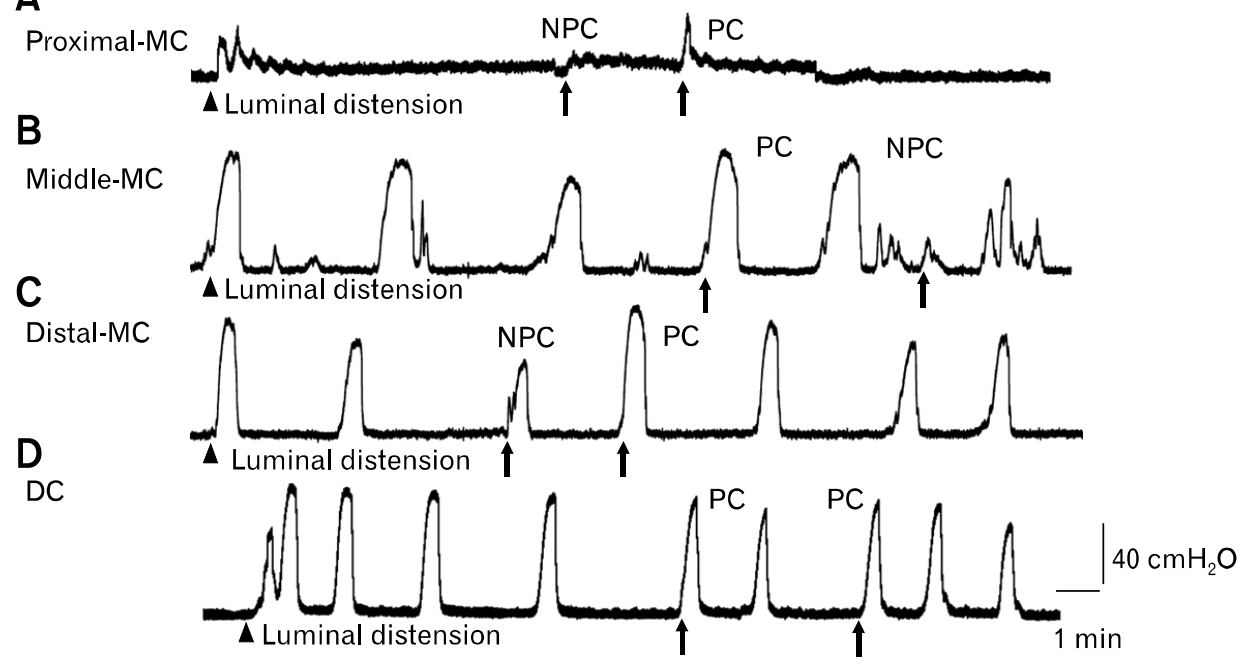

\section{E}

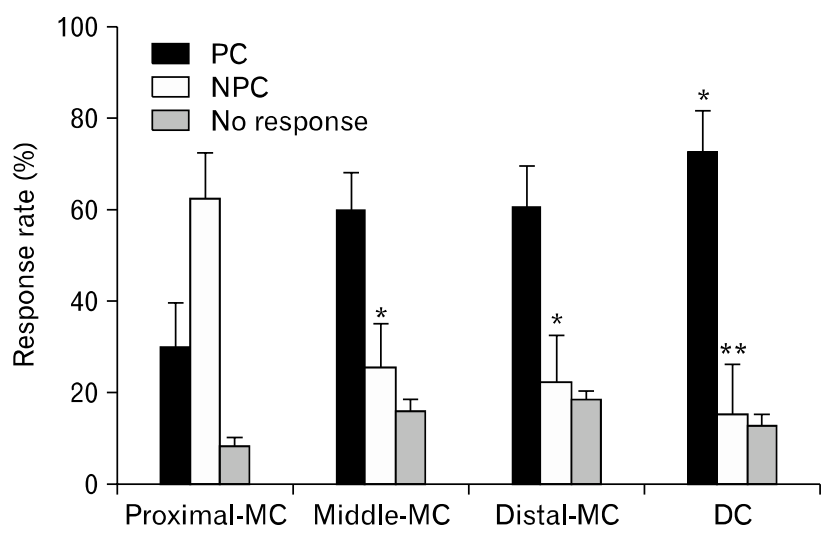

Figure 4. Responses to electrical field stimulation (EFS) applied at the oral end during the luminal distension in the guinea pig colonic segments. When EFS was applied after spontaneous peristaltic contraction (PC), EFS evoked PCs especially at the distal-mid colon and distal colon (C and D). In response to EFS, a significant increase of luminal pressure concomitant with $\mathrm{PC}$ was observed in $74.7 \%$ trials in the distal colon (D and $\mathrm{E})$. The incidence of non-peristaltic contractions (NPCs) and no responses were observed in $13.7 \%$ and $11.7 \%$, respectively, of the distal colon (48 studies of 6 tissues). The incidence of PCs was significantly increased in the distal colon, compared to that of the proximal-mid colon. In contrast, the incidence of NPCs was significantly lower in the middle-mid colon, distal-mid colon and distal colon, compared to that of proximal-mid colon (E) (45-60 studies of 6 tissues). Arrows indicate applications of EFS. MC, mid colon; DC, distal colon. ${ }^{*} P$ $<0.05,{ }^{*} P<0.01$ vs. proximal-mid colon. 

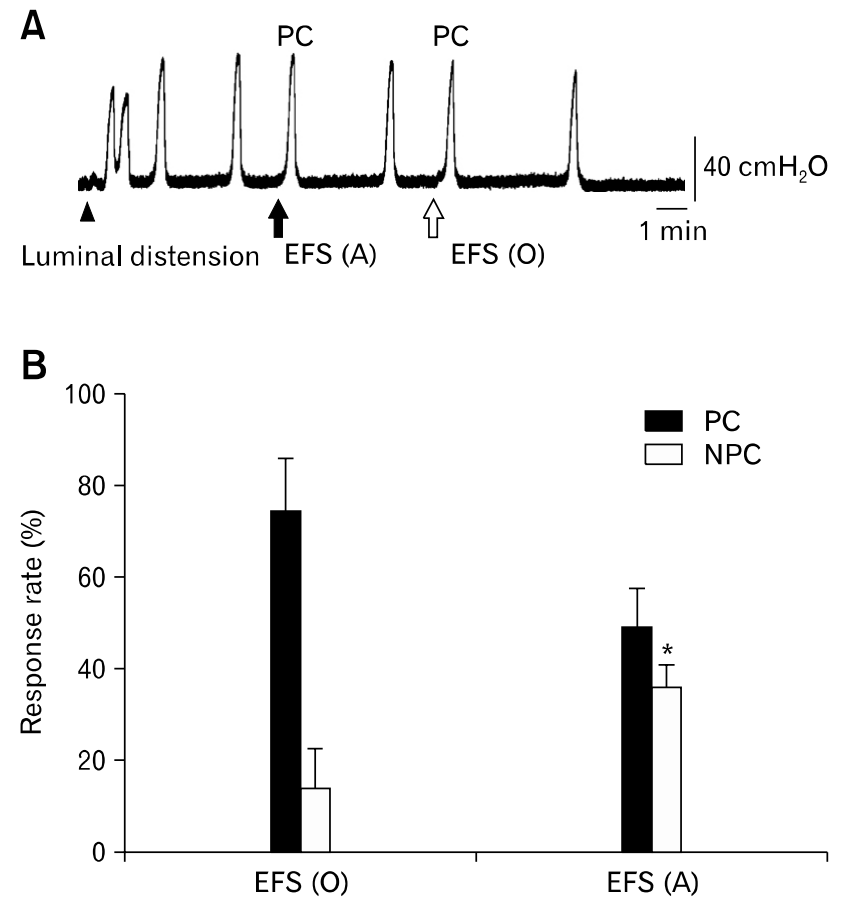

Figure 5. Responses to electrical field stimulation (EFS) at the oral and anal end of the distal colon. EFS-induced peristaltic contractions (PCs) were always oral-to-anal direction regardless of stimulation point and the mean amplitude was not significantly different between both stimulation points (A). Orally applied EFS tended to increase the incidence of PCs more than anally applied EFS $(P=0.06)$. The incidence of NPCs was significantly higher in response to anally applied EFS than orally applied EFS (B) $\left({ }^{*} P<0.05\right.$ vs. oral-EFS, $\left.\mathrm{n}=5\right)$. NPCs, non-peristaltic contractions.

sponse to 50 EFSs, $\mathrm{n}=6)$ of the guinea pig distal colon (20-24 studies of 5 tissues, $P<0.05$ ) (Fig. 5B).

Surprisingly, PCs were observed 3-10 seconds following oral-EFS (Video 1), while PCs were immediately observed following the application of anal-EFS (Video 2). EFSs on either side did not cause any retrograde propagation.

\section{Effect of L-NAME, Hexamethonium, Atropine and Tetrodotoxin on Electrical Field Stimula- tion-induced Peristaltic Reflex of the Distal Colon}

L-NAME $\left(10^{-4} \mathrm{M}\right)$ itself decreased the incidence of PCs to $26 \%$ and increased the incidence of NPCs to $70 \%$ in response to luminal distension $(\mathrm{n}=6)$ (Fig. 6A). When EFS was applied to the oral or anal end of the colon, non-coordinated NPCs were observed in the distal colon in the presence of L-NAME (Fig. 6A and Video 3).

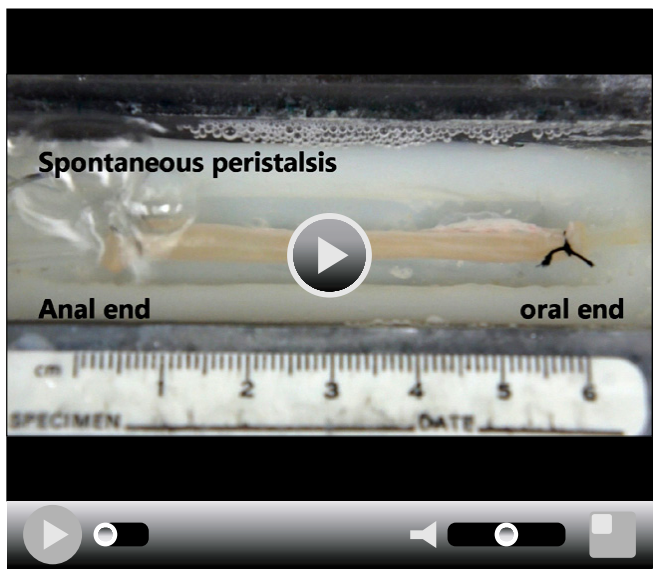

Video 1. Motor responses to electrical field stimulation (EFS) applied to the oral end of the guinea pig distal colon. Fifteen to 20 seconds after finishing spontaneous peristaltic reflex, EFS was applied to the oral end of the distal colon. EFS was able to induce peristalsis. There was a time lag (3-10 seconds) observed between the application of EFS and initiation of peritalsis. (Video clip is available at http://www.jnmjournal. org/journal/journal_view.html?year $=2013 \& \mathrm{vol}=019 \&$ num $=02 \&$ page $=192)$.

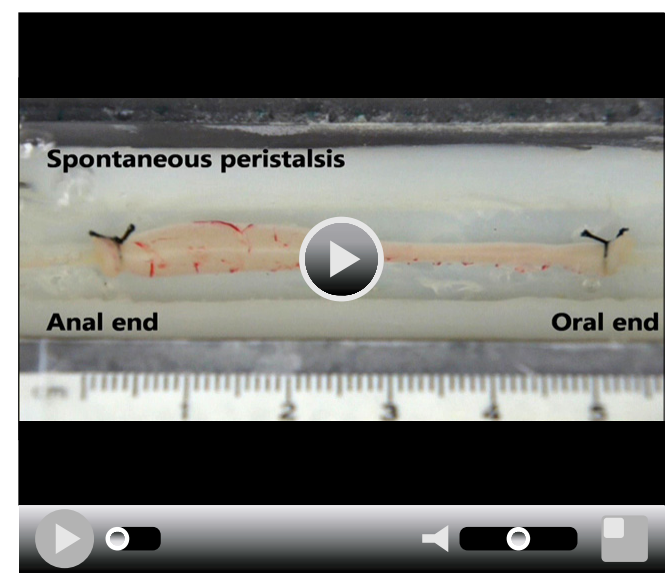

Video 2. Motor responses to electrical field stimulation (EFS) applied to the anal end of the guinea pig distal colon. In contrast to orally applied EFS, anal EFS stimulation was able to induce an immediate peristaltic reflex without any time lag. (Video clip is available at http://www. jnmjournal.org/journal/journal_view.html? year $=2013 \& \mathrm{vol}=$ 019\&num $=02 \&$ page $=192$ ).

When hexamethonium $\left(10^{-4} \mathrm{M}\right)$, atropine $\left(10^{-6} \mathrm{M}\right)$ or TTX $\left(10^{-6} \mathrm{M}\right)$, was added in the organ bath, intraluminal pressure increase in response to luminal distension was no longer observed. EFS failed to cause any PCs or NPCs in the presence of hexamethonium, atropine or TTX (Fig. 6B-D). 
A

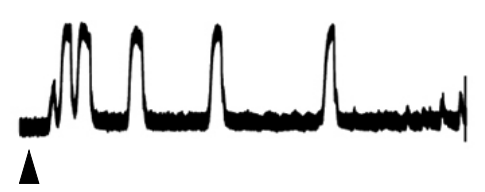

Luminal distension

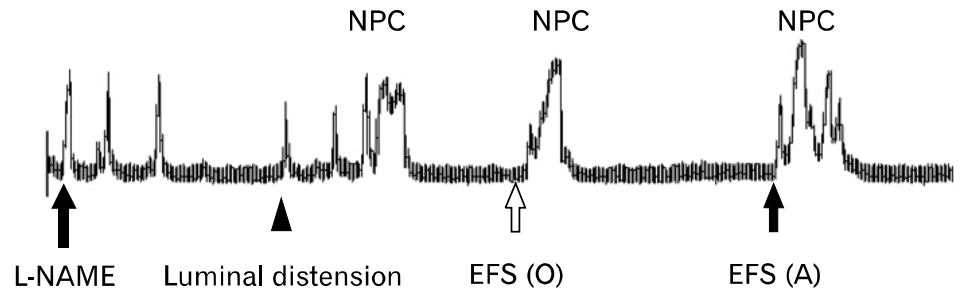

L-NAME Luminal distension EFS (O)
EFS (A)

B

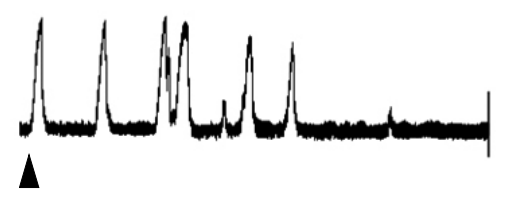

Luminal distension

Hexamethonium

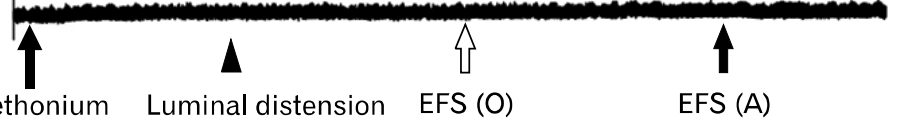

C

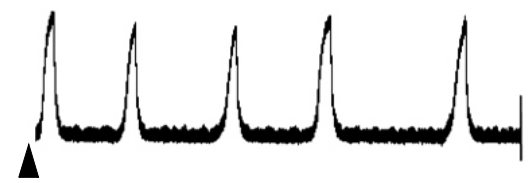

Luminal distension

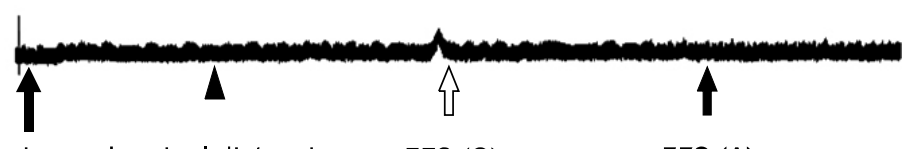

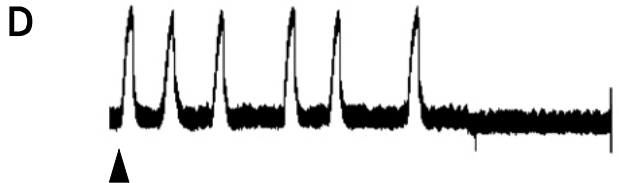

Luminal distension
Atropin

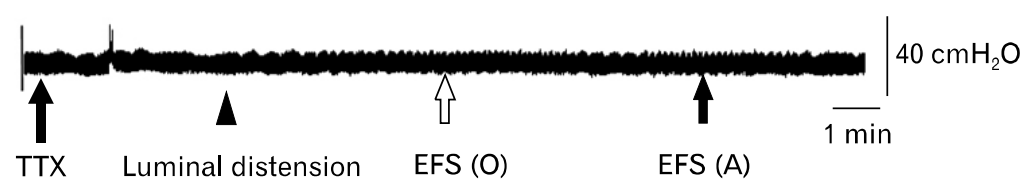

Figure 6. Responses to electrical field stimulation (EFS) at the oral and anal end of the distal colon in the presence of neural blocking agents. Administration of NG-nitro-L-arginine methyl ester (L-NAME) itself increased the incidence of non-peristaltic contractions (NPCs) to $70 \%$. When EFS was applied to the oral or anal end of the colon, non-coordinated NPCs were observed in the distal colon in the presence of L-NAME (A) (Video 3). In the presence of hexamethonium, atropine and and tetrodotoxin, intraluminal pressure increase in response to luminal infusion was no longer observed. Hexamethonium, atropine and TTX completely abolished the responses to EFS (B-D) ( $\mathrm{n}=6$ in each group). O, oral end; A, anal end.

\section{Discussion}

Small animals such as rats ${ }^{14,28}$ and guinea pigs ${ }^{15,16}$ have been widely used to study regional difference of the colonic motility. It has been shown that the inserted pellets are well advanced distally in in vitro preparations of the guinea pig colon, but not rat colon. ${ }^{29-31}$ Our preliminary study also showed that the rat distal colon failed to propel fecal pellets in vitro (unpublished observations). However, the mechanisms of the different motility responses to inserted pellets between the two animals have not been fully explained.
Peristaltic reflexes in response to stretch and mucosal stimulation are mediated via both extrinsic and intrinsic neurons of the rat colon. ${ }^{32}$ In contrast, both stretch and mucosal reflexes are mediated via intrinsic neurons of the guinea pig colon. ${ }^{33}$ Therefore, it is conceivable, that due to the lack of extrinsic innervation of the rat colon, the pellet-stimulated motor responses are less observed in in vitro preparations. The intrinsic neural pathway mediating peristaltic reflex may not be well developed in the rat distal colon.

We have previously shown that the pelvic nerve regulates colorectal contractions via muscarinic receptors, while the hypogastric nerve regulates colorectal relaxations via beta-adreno- 


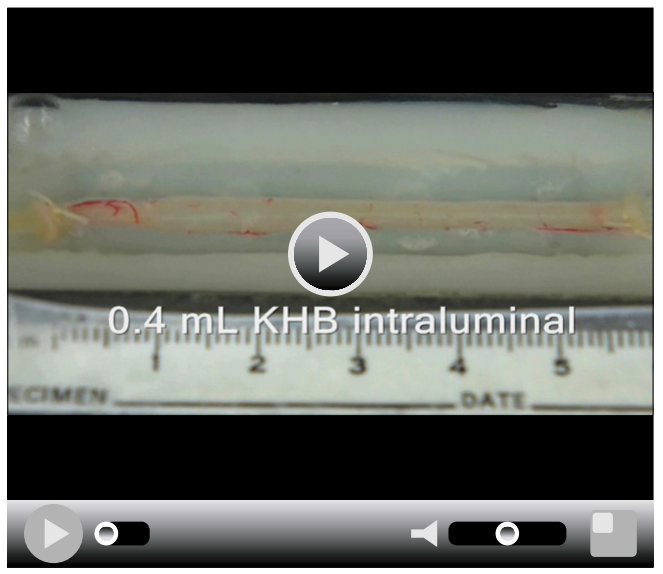

Video 3. Motor responses to orally and anally applied electrical field stimulation (EFS) in the presence of NG-nitro-L-arginine methyl ester (L-NAME) of the guinea pig distal colon. L-NAME itself increased the incidence of non-peristaltic contractions (NPCs). When EFS was applied to the oral or anal end of the colon, non-coordinated NPCs were observed. (Video clip is available at http://www.jnmjournal.org/journal/ journal_view.html?year $=2013 \& \mathrm{vol}=019 \&$ num $=02 \&$ page $=192$ ). KHB, Krebs-Henseleit buffer.

ceptors in rats. ${ }^{34}$ We also showed that the pelvic nerve dissection significantly impaired colonic transit and colonic motility in rats. ${ }^{35,36}$ Thus, it is likely that extrinsic innervation, rather than intrinsic innervation, plays a major role in regulating colonic peristalsis of the rat colon. To study the mechanism of intrinsic neurons in mediating peristaltic reflex, we utilized guinea pig colon.

Previous studies have investigated the mechanisms underlying the regional differences in colonic motility. ${ }^{14,15}$ We have previously demonstrated in a rat model that the number of NOS containing neurons and the NOS activity were increased in the myenteric plexus of the proximal colon compared with the distal colon, which was consistent with the observations of lower tone, accommodation and mixing function of the proximal colon. ${ }^{14}$ The distal colon is considered to have greater contractile responses to EFS, probably because of the enhanced cholinergic pathway in the myenteric plexus. ${ }^{15}$

The proximal colon has a greater capacity. ${ }^{37}$ In addition to these characteristics, the motor activity in the proximal part of the middle colon, induced by fluid infusion, showed frequent nonpropagating, non-coordinated local contractions rather than PCs. These contractions were detected irregularly and repeatedly at different points in the isolated colonic segment, which were suitable for mixing and absorptive functions. Moreover, the mean amplitude of PCs in the proximal portion of the middle colon was approximately half of that in the distal colon. The diame- ter of the proximal colon was reduced by $29 \%$ compared to resting diameter during the peristaltic contraction, while PC of the distal colon occluded the lumen. ${ }^{16}$ These features indicate that the motor patterns of the proximal part of the colon are not focused on emptying but rather slowly pushing the contents. On the other hand, the distal colon is of narrower caliber and has a thicker external muscular layer, reflecting the propulsive function of this region. ${ }^{37}$

The high propulsion velocity of a natural pellet might be influenced by this anatomical advantage. In our current study, the distal colon displayed active and potent motor patterns that are involved in physiological emptying of the fecal contents. The recording of pressure changes in response to fluid distension is not identical with normal physiological condition because it keeps the colonic segment evenly distended due to a closed circuit for a certain period (10 minutes). Although the distal colonic segment was continuously stimulated by the luminal distension, there was a resting period between successive PCs. Spontaneous PCs were produced every 90-100 seconds in the distal colon during the fluid distension. This suggests that underlying factors trigger peristaltic reflex.

Electrical field stimulation can activate enteric nervous system and/or muscle cells directly or both. It is generally accepted that the enteric neurons can be activated by a pulse shorter than 1 $\mathrm{ms}$, whereas EFS with long pulse ( $>50 \mathrm{~ms}$ ) can depolarize muscle cells directly. ${ }^{38}$ Atropine blocked propulsion stimulated by short pulses $(0.3 \mathrm{~ms})$, but not long pulses $(30 \mathrm{~ms})$ in the rat distal colon. ${ }^{21}$ Therefore, it is likely that stimulation with short pulses induces colonic contraction through the activation of cholinergic transmission, whereas long pulse stimulation induces myogenic contraction.

EFS-induced motor responses were completely abolished by TTX, suggesting that the intramural neurons are involved in mediating peristaltic reflex. Immediately after EFS, a significant increase in the luminal pressure increase concomitant with PC was frequently observed in the distal colon and the distal portion of the middle colon. A PC was inducible by the application of EFS even in a resting period, indicating that intrinsic triggers to initiate peristaltic reflex are neurogenic.

Previous studies showed that colonic motor responses to EFS were always localized and were not able to propel intraluminal contents over a distance in rats ${ }^{21}$ and dogs. ${ }^{22}$ In contrast, when EFS was applied sequentially from the proximal range to distal range, aborad contractions were observed in the dog distal colon. ${ }^{22}$ However, our current study of guinea pig distal colon 
showed that a single EFS (oral or anal) initiated PCs which may propel intraluminal contents. It is not clear whether different neuronal regulatory mechanisms are involved in mediating colonic motility among dogs, rats, and guinea pigs.

When electrical stimulation was applied sequentially from the distal range to proximal range, orad direction contractions (anti-peristalsis) were produced in the distal colon of dogs. ${ }^{22}$ Contrary to our original expectation, EFS applied to the anal end produced propagating peristaltic contractions (PCs) rather than anti-peristalsis in the guinea pig distal colon. We could observe the time lag (3-10 seconds) between the application of EFS and initiation of PCs when EFS was applied to the oral end. In contrast, this delay was not observed in response to EFS applied anally.

It is well established that the colonic peristaltic reflex is mediated by the release of 5-hydroxytryptamine (5-HT) from enterochromaffin cells. ${ }^{32} 5$-HT acts through $5-\mathrm{HT}_{4}$ and $5-\mathrm{HT}_{3}$ receptors to activate intramural sensory neurons that release calcitonin gene-related peptide. ${ }^{39}$ The presence of fecal pellet triggers the release of 5-HT, which acts via both $5-\mathrm{HT}_{3}$ and $5-\mathrm{HT}_{4}$ receptors to regulate propulsive activity of the guinea pig colon. ${ }^{30}$ Others showed that hexamethonium abolished the peristaltic reflex, suggesting the involvement of nicotinic receptors in the initiation of peristaltic reflex of the guinea pig colon. ${ }^{31}$

Mucosal and muscle stimulation initiates peristalsis via ascending contractions and descending relaxations. Ascending contractions are mainly mediated via acetylcholine ${ }^{40}$ and substance $P$, while descending relaxations are mainly mediated via nitric oxide (NO), vasoactive intestinal polypeptide and pituitary adenylate cyclase-activating peptide. ${ }^{41-43}$ Especially, released acetylcholine and NO from the myenteric plexus play a crucial role in mediating peristalsis.

We have recently shown that $5-\mathrm{HT}_{3}$ and $5-\mathrm{HT}_{4}$ receptors promote colonic peristalsis via different mechanisms in guinea pigs. ${ }^{44}$ In the current study, we focused the possible role of muscarinic receptors, nicotinic receptors, and NO in mediating peristaltic reflex of the guinea pig colon.

In the presence of L-NAME, the incidence of PCs was reduced while the incidence of NPCs was increased in the distal colon. When EFS was applied to the oral or anal end of the colon, non-coordinated NPCs were observed in the presence of L-NAME. This suggests that neuronal NO plays a crucial role in mediating peristalsis.

Hexamethonium and atropine abolished intraluminal pressure increase in response to luminal infusion. EFS failed to cause any PCs or NPCs in the presence of hexamethonium, and atropine. This suggests that both of muscarinic and nicotinic receptors are involved in mediating peristalsis of the guinea pig colon.

Interestingly, recent studies have shown that fecal pellet-induced peristalsis in guinea-pig distal colon does not require endogenous activation of nicotinic, $5-\mathrm{HT}_{3}, \mathrm{NK}_{3}$ or $\mathrm{P}_{2}$ receptors, ${ }^{45}$ and the nature of the neurotransmitters required for peristalsis are not fully understood. It is conceivable that orally applied EFS may stimulate both excitatory and inhibitory neurotransmission, while anally applied EFS may mainly stimulate excitatory neurotransmission. It is likely that the activation of ascending excitatory neurons by anal-EFS produced immediate peristaltic contractions, commencing at the oral end. The incidence of NPCs in response to orally applied EFS was significantly lower than that seen in response to anally applied EFS, suggesting that the neural activation at the oral side, rather than anal side, may regulate the normal peristalsis. However, the mechanism of peristalsis is not fully understood and its regulation remains to be studied.

The guinea pig distal colon can be an ideal model for the study of peristalsis due to high incidence of NPs. This study may help us understand colonic motility by demonstrating the unique motor pattern of each colonic part for studying colonic motor activity including peristalsis.

\section{References}

1. Vantrappen G, Janssens J, Hellemans J, Ghoos Y. The interdigestive motor complex of normal subjects and patients with bacterial overgrowth of the small intestine. J Clin Invest 1977;59:1158-1166.

2. Itoh Z. Motilin and clinical application. Peptides 1997;18:593-608.

3. Ariga H, Tsukamoto K, Chen C, Mantyh C, Pappas TN, Takahashi T. Endogenous acyl ghrelin is involved in mediating spontaneous phase III-like contractions of the rat stomach. Neurogastroenterol Motil 2007;19:675-680.

4. Zheng J, Ariga H, Taniguchi H, Ludwig K, Takahashi T. Ghrelin regulates gastric phase III-like contractions in freely moving conscious mice. Neurogastroenterol Motil 2009;21:78-84.

5. Phillips SF. Functions of the large bowel: an overview. Scand J Gastroenterol 1984;19(suppl 93):1-12.

6. Sleisenger MH, Feldman M, Friedman LS, Brandt LJ. Sleisenger and Fordtran's gastrointestinal and liver disease: pathophysiology, diagnosis, management. 9th ed. Philadelphia: Saunders/Elsevier, 2010:1659-1674.

7. Barrett KE. Gastrointestinal physiology. New York: Lange Medical Books/McGraw-Hill, Medical Pub. Division 2006:295.

8. Sarna SK, Condon R, Cowles V. Colonic migrating and nonmigrating motor complexes in dogs. Am J Physiol 1984;246(4 Pt 1): 
G355- G360.

9. Sarna SK. Myoelectric correlates of colonic motor complexes and contractile activity. Am J Physiol 1986;250(2 Pt 1):G213-G220.

10. Sethi AK, Sarna SK. Contractile mechanisms of canine colonic propulsion. Am J Physiol 1995;268(3 Pt 1):G530-G538.

11. Cherbut C, Ferrier L, Rozé C, et al. Short-chain fatty acids modify colonic motility through nerves and polypeptide $\mathrm{YY}$ release in the rat. Am J Physiol 1998;275 (6 Pt 1): G1415-G1422.

12. Hennig GW, Gregory S, Brookes SJ, Costa M. Non-peristaltic patterns of motor activity in the guinea-pig proximal colon. Neurogastroenterol Motil 2010;22:e207-e217.

13. Ritchie JA. Colonic motor activity and bowel function. II. Distribution and incidence of motor activity at rest and after food and carbachol. Gut 1968;9:502-511.

14. Takahashi T, Owyang C. Regional differences in the nitrergic innervation between the proximal and the distal colon in rats. Gastroenterology 1998;115:1504-1512.

15. Hasler WL, Kurosawa S, Chung OY. Regional cholinergic differences between distal and proximal colonic myenteric plexus. Am J Physiol 1990;258(3 Pt 1):G404-G410.

16. D'Antona G, Hennig GW, Costa M, Humphreys CM, Brookes SJ. Analysis of motor patterns in the isolated guinea-pig large intestine by spatio-temporal maps. Neurogastroenterol Motil 2001;13:483-492.

17. Sundler F, Ekblad E, Håkanson R. Projections of enteric peptide-containing neurons in the rat. Arch Histol Cytol 1989;52(suppl):181-189.

18. Timmermans JP, Adriaensen D, Cornelissen W, Scheuermann DW. Structural organization and neuropeptide distribution in the mammalian enteric nervous system, with special attention to those components involved in mucosal reflexes. Comp Biochem Physiol A Physiol 1997;118:331-340.

19. Cunningham SM, Hirai K, Mihara S, Lees GM. Electrophysiological characteristics of submucosal neurones in the proximal colon of guinea-pigs: comparisons with caecum and descending colon. Exp Physiol 1997;82:859-870.

20. Browning KN, Cunningham SM, Duncan L, Timmermans J, Lees GM. Regional differences in the sympathetic innervation of the guinea pig large intestine by neuropeptide $\mathrm{Y}$ - and tyrosine hydroxylaseimmunoreactive nerves of divergent extrinsic origin. J Comp Neurol 1999;410:515-530.

21. Sevcencu C, Rijkhoff NJ, Sinkjaer T. Muscular vs. neural activation in propulsion induced by electrical stimulation in the descending colon of rats. Neuromodulation 2005;8:131-140.

22. Amaris MA, Rashev PZ, Mintchev MP, Bowes KL. Microprocessor controlled movement of solid colonic content using sequential neural electrical stimulation. Gut 2002;50:475-479.

23. Yoneda S, Kadowaki M, Kuramoto H, Fukui H, Takaki M. Enhanced colonic peristalsis by impairment of nitrergic enteric neurons in spontaneously diabetic rats. Auton Neurosci 2001;92: $65-71$.

24. Gribovskaja-Rupp I, Takahashi T, Ridolfi T, Kosinski L, Ludwig $\mathrm{K}$. Upregulation of mucosal 5-HT3 receptors is involved in restoration of colonic transit after pelvic nerve transection. Neurogastroenterol Motil 2012;24:472-478.e218.

25. Hosoda K, Takahashi T, Fujino MA, Owyang C. Inhibitory effects of nitric oxide donors on nitric oxide synthesis in rat gastric myenteric plexus. J Pharmacol Exp Ther 1998;286:1222-1230.
26. Takahashi T, Owyang C. Vagal control of nitric oxide and vasoactive intestinal polypeptide release in the regulation of gastric relaxation in rat. J Physiol 1995;484:481-492.

27. Takahashi T, Owyang C. Characterization of vagal pathways mediating gastric accommodation reflex in rats. J Physiol 1997;504(Pt 2): 479-488.

28. Alberti E, Mikkelsen HB, Larsen JO, Jiménez M. Motility patterns and distribution of interstitial cells of Cajal and nitrergic neurons in the proximal, mid- and distal-colon of the rat. Neurogastroenterol Motil 2005;17:133-147.

29. Foxx-Orenstein AE, Jin JG, Grider JR. 5-HT4 receptor agonists and delta-opioid receptor antagonists act synergistically to stimulate colonic propulsion. Am J Physiol 1998;275(5 Pt 1):G979-G983.

30. Jin JG, Foxx-Orenstein AE, Grider JR. Propulsion in guinea pig colon induced by 5-hydroxytryptamine (HT) via 5-HT4 and 5-HT3 receptors. J Pharmacol Exp Ther 1999;288:93-97.

31. Kadowaki M, Wade PR, Gershon MD. Participation of 5-HT3, 5-HT4, and nicotinic receptors in the peristaltic reflex of guinea pig distal colon. Am J Physiol 1996;271(5 Pt 1):G849-G857.

32. Grider JR, Jin JG. Distinct populations of sensory neurons mediate the peristaltic reflex elicited by muscle stretch and mucosal stimulation. J Neurosci 1994;14(5 Pt 1):2854-2860.

33. Spencer NJ, Smith TK. Mechanosensory S-neurons rather than AH-neurons appear to generate a rhythmic motor pattern in guinea-pig distal colon. J Physiol 2004;558(Pt 2):577-596.

34. Tong WD, Ridolfi TJ, Kosinski L, Ludwig K, Takahashi T. Effects of autonomic nerve stimulation on colorectal motility in rats. Neurogastroenterol Motil 2010;22:688-693.

35. Ridolfi TJ, Tong WD, Takahashi T, Kosinski L, Ludwig KA. Sympathetic and parasympathetic regulation of rectal motility in rats. J Gastrointest Surg 2009;13:2027-2033; discussion 2033.

36. Ridolfi T, Tong W, Kosinski L, Takahashi T, Ludwig K. Recovery of colonic transit following extrinsic nerve damage in rats. Scand J Gastroenterol 2011;46:678-683.

37. Wattchow D, Brookes S, Murphy E, Carbone S, de Fontgalland D, Costa M. Regional variation in the neurochemical coding of the myenteric plexus of the human colon and changes in patients with slow transit constipation. Neurogastroenterol Motil 2008;20:12981305 .

38. Moritz A, Grundfest-Broniatowski S, Ilyes L, et al. Electrical pulse train and single pulse stimulation of the small intestine: acute and chronic studies in the dog. Artif Organs 1989;13:116-122.

39. Grider JR. CGRP as a transmitter in the sensory pathway mediating peristaltic reflex. Am J Physiol 1994;266(6 Pt 1):G1139-G1145.

40. Timmermans JP, Barbiers M, Scheuermann DW, et al. Distribution pattern, neurochemical features and projections of nitrergic neurons in the pig small intestine. Ann Anat 1994;176:515-525.

41. Foxx-Orenstein AE, Grider JR. Regulation of colonic propulsion by enteric excitatory and inhibitory neurotransmitters. Am J Physiol 1996;271(3 Pt 1):G433-G437.

42. Grider JR. Interplay of VIP and nitric oxide in regulation of the descending relaxation phase of peristalsis. Am J Physiol 1993;264(2 Pt 1): G334-G340.

43. Murthy KS, Jin JG, Grider JR, Makhlouf GM. Characterization of PACAP receptors and signaling pathways in rabbit gastric muscle cells. Am J Physiol 1997;272(6 Pt 1):G1391-G1399. 
44. Gribovskaja-Rupp I, Kwak J, Takahashi T, Ludwig K. 5-HT3 and 5-HT4 receptors promote colonic peristalsis via different mechanisms in guinea pigs [abstract]. Digestive Diseases Week 2012:465.

45. Nicholas S, Spencer NJ. Peristalsis and fecal pellet propulsion do not require nicotinic, purinergic, 5-HT3, or NK3 receptors in isolated guinea pig distal colon. Am J Physiol Gastrointest Liver Physiol 2010;298:G952-G961. 